\title{
PML-RARA can increase hematopoietic self-renewal without causing a myeloproliferative disease in mice
}

\author{
John S. Welch, Wenlin Yuan, and Timothy J. Ley \\ Section of Stem Cell Biology, Division of Oncology, Departments of Medicine and Genetics, \\ Washington University School of Medicine, St. Louis, Missouri, USA.
}

\begin{abstract}
Acute promyelocytic leukemia (APL) is characterized by the $t(15 ; 17)$ translocation that generates the fusion protein promyelocytic leukemia-retinoic acid receptor $\alpha$ (PML-RARA) in nearly all cases. Multiple prior mouse models of APL constitutively express $P M L-R A R A$ from a variety of non-Pml loci. Typically, all animals develop a myeloproliferative disease, followed by leukemia in a subset of animals after a long latent period. In contrast, human APL is not associated with an antecedent stage of myeloproliferation. To address this discrepancy, we have generated a system whereby $P M L-R A R A$ expression is somatically acquired from the mouse $P m l$ locus in the context of $P \mathrm{ml}$ haploinsufficiency. We found that physiologic PML-RARA expression was sufficient to direct a hematopoietic progenitor self-renewal program in vitro and in vivo. However, this expansion was not associated with evidence of myeloproliferation, more accurately reflecting the clinical presentation of human APL. Thus, at physiologic doses, $P M L-R A R A$ primarily acts to increase hematopoietic progenitor self-renewal, expanding a population of cells that are susceptible to acquiring secondary mutations that cause progression to leukemia. This mouse model provides a platform for more accurately dissecting the early events in APL pathogenesis.
\end{abstract}

\section{Introduction}

More than 95\% of acute promyelocytic leukemia (APL; FAB-M3) cases are associated with the recurrent $\mathrm{t}(15 ; 17)(\mathrm{q} 22: \mathrm{q} 11)$ translocation, which results in the expression of a fusion transcript promyelocytic leukemia-retinoic acid receptor $\alpha(P M L-R A R A)$, inconsistent expression of a reciprocal fusion product (RARA-PML), and haploinsufficiency for both $P M L$ and $R A R A(1,2)$. Although APL has served as an important model disease for understanding leukemogenic pathways directed by a specific translocation, the precise mechanisms by which $t(15 ; 17)$ initiates leukemia remain unknown.

Several mouse models have been used to study the mechanisms by which PML-RARA causes transformation (reviewed in refs. 3, 4). These models have used viral transduction, transgenic, and knockin strategies; regulatory sequences from the MRP8 gene (also known as S100 calcium binding protein A8 [S100A8]) and human and murine cathepsin G (CTSG and Ctsg, respectively) genes have been used to direct the expression of PML-RARA to the myeloid compartment (5-8), where can cause a myeloproliferative disease and APL after a long latent period.

However, there are many issues with existing mouse models of APL that must be addressed to fully understand how PML-RARA works. (a) The dose of PML-RARA is known to be important for AML penetrance, but no model to our knowledge has yet expressed $P M L-R A R A$ from the endogenous $P m l$ locus. This has not previously been attempted, since $P m l$ is ubiquitously expressed, and since widespread expression of PML-RARA during development causes embryonic lethality $(9,10)$. (b) $\mathrm{t}(15 ; 17)$ is a somatically acquired event, but all prior models have used constitutive PML-RARA expression, raising the possibility that hematopoietic cells may have adapted to tolerate $P M L-R A R A$ expression during embryo-

Conflict of interest: The authors have declared that no conflict of interest exists. Citation for this article: J Clin Invest. 2011;121(4):1636-1645. doi:10.1172/JCI42953. genesis and/or early adult life. (c) Although $P m l$ haploinsufficiency has been shown to contribute to APL pathogenesis, this has not been routinely integrated into mouse models (11).

To better define the initial effects of PML-RARA on hematopoiesis, we have developed a murine model (mPML-PRflox mice). We used homologous recombination to express PML-RARA under the control of the murine $P m l$ locus and used Cre-Lox technology to prevent PML-RARA expression prior to Cre recombination (to prevent embryonic lethality). This model recapitulates multiple elements of the pathologic $t(15 ; 17)$ translocation that have been absent in prior murine models of APL (i.e., $P m l$ locus-appropriate expression of PML-RARA, Pml haploinsufficiency, and somatic acquisition of oncoprotein expression in adult animals).

In this report, we show that this model differs substantially from all previous ones and allows interrogation of initial PML-RARA effects on hematopoiesis after somatic acquisition of expression. When an ERT2-Cre cassette (driven by a chicken $\beta$-actin/CMV promoter/enhancer) is activated by tamoxifen in adult mice, mPML-PRflox is floxed and induces an inappropriate hematopoietic self-renewal program: floxed mPML-PRflox (designated mPML-fPR) bone marrow CFUs could be serially replated for 6 or more weeks ex vivo and a progressive expansion of mPMLfPR-positive hematopoietic cells occurred in vivo. Remarkably, this expansion occurred in the absence of concurrent myeloproliferation and was observed in both myeloid and lymphoid compartments after competitive transplantation. These studies show that PML-RARA alters self-renewal programs but does not alter homeostatic feedback mechanisms that control the size of the preleukemic myeloid compartment itself.

\section{Results}

$P M L$ and RARA expression in normal and leukemic human bone marrow cells. Because many translocations result in aberrant expression of at least one nearby gene (12), we evaluated the expression of both 
A
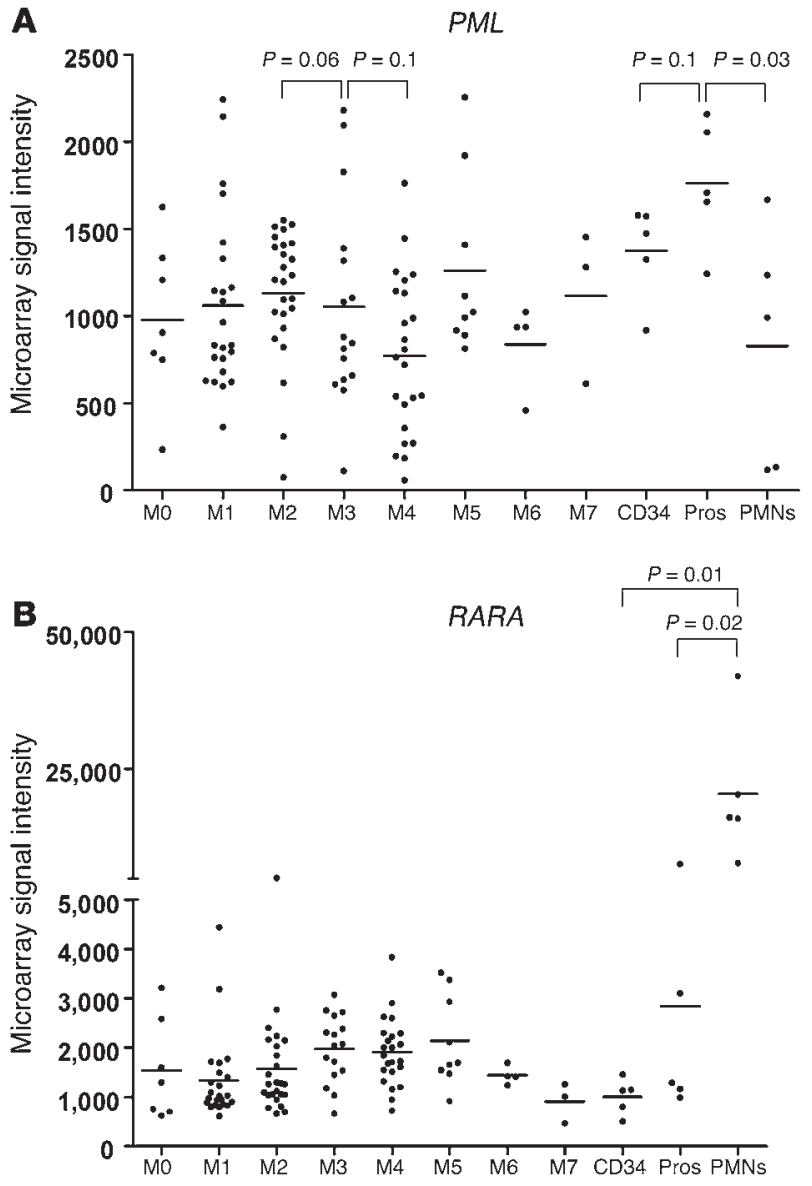

$P M L$ and RARA across a wide range of AML patients with various FAB subtypes and in 5 normal volunteers, after flow sorting for $\mathrm{CD}^{2} 4^{+}$, promyelocyte, and neutrophil (PMN) populations (13). We found no significant alteration in the expression of $P M L$ or $R A R A$ in patients with $\mathrm{t}(15 ; 17)$ compared with those with non-FAB-M3 AML (Figure 1). This suggests that $\mathrm{t}(15 ; 17)$ does not influence hematopoiesis by grossly dysregulating the expression of either $P M L$ or RARA. These data also confirmed the prior observation that RARA expression is markedly upregulated during terminal myeloid maturation (Figure $1 \mathrm{~B}$ and ref. 14).

Generation of mice with the MPML-PRflox allele. To more closely recapitulate the effects of dose-appropriate expression and somatic acquisition of PML-RARA on hematopoiesis, we used homologous recombination to insert a loxP-PGKneo-stop-loxP$P M L-R A R A$ cDNA into the murine $P m l 5$ ' untranslated region (designated mPML-PRflox) of a C57BL/6-derived (B6-derived) ES cell line (B6-Blu), which was then used to generate B6 mice with this mutant allele (Figure 2A). Five stop codons positioned across all 3 reading frames were inserted downstream from the PGK-neo cassette to prevent expression of PML-RARA prior to Cre-mediated recombination. mPML-PRflox mice are viable and display normal Mendelian inheritance and normal peripheral blood cell counts (data not shown). The Cre-recombined allele expresses PML-RARA mRNA and protein and is referred to hereafter as mPML-fPR.

We first assessed the effect of the mPML-PRflox allele on Pml expression using Western blotting (Supplemental Figure 1A). As expected, we observed a decrease in Pml protein in $m P M L$ -

\section{Figure 1}

Expression profiling. (A and $\mathbf{B}$ ) Expression array profile of $(\mathbf{A})$ human $P M L$ and (B) human RARA in 111 patients with indicated $F A B-A M L$ and 5 flow-sorted normal controls. CD34, CD34+ bone marrow cells; Pros, promyelocytes. Individual data points represent results from individual patients. Horizontal bars are the median value.

$P R f_{l o x^{+/-}}$spleen cells and a complete loss of Pml protein in $m P M L$ $P R f l o x^{+/+}$spleen cells. These data confirm that mPML-PRflox results in a null mutation in $P m l$ and that heterozygosity creates haploinsufficiency for $P m l$.

We crossed mPML-PRflox mice (made in a B6 ES cell line and maintained in the $\mathrm{B} 6$ strain) with several different Cre-expressing mouse lines that were also made in or backcrossed to the $\mathrm{B} 6$ background. We first crossed mPML-PRflox mice to Mx-Cre mice but did not observe

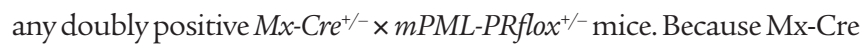
is expressed at low levels even without interferon activation and $\mathrm{Pml}$ is expressed widely during embryogenesis, we suspected that these mice died during embryogenesis because of $\mathrm{Mx}$-Cre "leakiness" $(9,15,16)$. To confirm this result, we crossed male $\mathrm{CMV}$-Cre $\mathrm{C}^{+/-}$ mice with female $m P M L-P R f^{+} x^{+/-}$mice. We observed 7 out of $16 \mathrm{CMV}-\mathrm{Cre}^{-/-} \times \mathrm{mPML}-\mathrm{PR}$ flox $^{+/-}$male pups but 0 out of 13 $\mathrm{CMV}-\mathrm{Cre}^{+/-} \times m P M L-P R$ flox $^{+/-}$female pups (CMV-Cre is X-linked, and the female pups are obligate Cre heterozygotes) (Figure $2 \mathrm{~B}$ ). We further evaluated 51 E13.5 embryos from a total of 6 litters of $\mathrm{CMV}-\mathrm{Cre} \mathrm{C}^{+/-} \times \mathrm{mPML}-\mathrm{PR} \mathrm{flox}^{+/-}$intercrosses. Of these, 26 embryos were $\mathrm{CMV}-\mathrm{Cre}^{+/-}, 16$ embryos were $m P M L-P R \mathrm{flox}^{+/}$, and 0 embryos were $C M V-C r e^{+/-} \times m P M L-P R f l o x^{+/-}$. No resorbed embryos were observed in any of the 6 litters. This confirms that the floxed mPML-fPR allele causes early embryonic lethality when PML-RARA is widely expressed (consistent with prior observations that PML-RARA can cause cellular toxicity) (17-19) and provides strong evidence that $P M L-R A R A$ is functional when it is activated by Cre-recombinase in this locus.

In contrast, Cre cassettes with more restricted expression patterns could be successfully intercrossed; doubly heterozygous mice containing the LysM-Cre or the ERT2-Cre allele survived to adulthood with normal blood counts and without gross phenotypes (LysM-Cre expression is limited to differentiated myeloid cells, and ERT2-Cre is active only after tamoxifen treatment) (20-22). Due to previously described cellular and embryonic toxicity from $P M L-R A R A$ expression, we were surprised that ERT2$\mathrm{Cr}^{+/-} \times m P M L-P R$ flox $^{+/-}$mice tolerated i.p. tamoxifen administration without evidence of gross toxicity $(17-19,22)$.

$P M L-R A R A$ mRNA could be detected using qRT-PCR in LysM$\mathrm{Cre}^{+/-} \times m P M L-P R$ flox $^{+/-}$spleen cells and also in ERT2-Cre ${ }^{+/-} \times$ $m P M L-P R f l o x^{+/-}$bone marrow cells after tamoxifen treatment (Supplemental Figure 1, B and C; supplemental material available online with this article; doi:10.1172/JCI42953DS1). We compared this expression to our previous model of APL, in which the same $P M L-R A R A$ cDNA is expressed from the Ctsg locus using a knockin strategy (herein referred to as $\mathrm{mCG}-\mathrm{PR}$ mice), which results in constitutive PML-RARA expression in hematopoietic progenitors, promyelocytes, and PMNs (peak expression is in promyelocytes) (7). The mPML-fPR allele yielded lower PML-RARA mRNA expression, consistent with the fact that the $P m l$ gene is expressed at much lower levels than the Ctsg gene during normal hematopoiesis (Welch, et al., unpublished observations). However, PML-RARA mRNA was much more abundant in the leukemic spleen cells of a LysM-Cre ${ }^{+/-} \times m P M L-P R f_{l o x}{ }^{+/-}$mouse, perhaps because virtually all cells in the sample contained an active mPML-fPR allele (Supple- 
A

Targeting strategy: mPML-PRflox

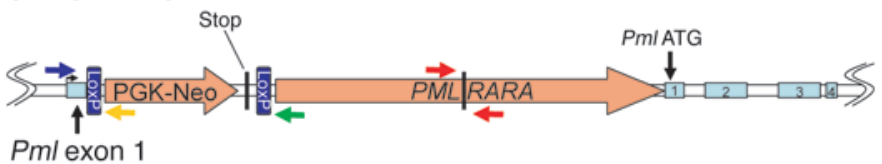

B

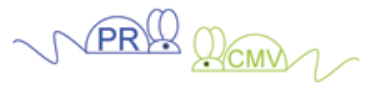

mPML-PRflox $\times$ CMV-Cre

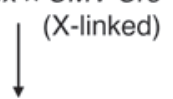

\begin{tabular}{|c|c|c|}
\hline \multicolumn{3}{|c|}{$\underline{m P M L-P R f l o x^{+/-}}$WT } \\
\hline $\begin{array}{l}\text { Males } \\
\left(\mathrm{CMV}-\mathrm{Cre}^{-/-}\right)\end{array}$ & 7 & 9 \\
\hline $\begin{array}{l}\text { Females } \\
\left(\mathrm{CMV}-\mathrm{Cre}^{+/-}\right)\end{array}$ & 0 & 13 \\
\hline
\end{tabular}

C

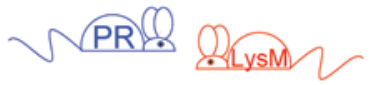

mPML-PRflox $\times$ LysM-Cre

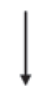

Expression of $\mathrm{PR}$ in spleen cells $\mathrm{Pml}$ microstipple pattern in PMNs No in vitro self-renewal Rare leukemia with APL features
D

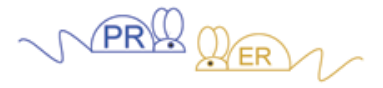

$m P M L-P R$ flox $\times$ ERT2-Cre<smiles>CCCCCCC</smiles>

Pml microstipple pattern In vitro self-renewal In vitro clonal expansion In vivo clonal expansion

\section{Figure 2}

mPML-PRflox targeting strategy and the result of intercrosses with CMV-Cre, LysMCre, and ERT2-Cre mice. (A) Targeting strategy. Rectangles represent exons. (B) The results of intercrosses with $\mathrm{MPML}$ PRflox $\times$ CMV-Cre, (C) mPML-PRflox $\times$ LysM-Cre, and (D) mPML-PRflox $\times$ ERT2Cre mice are shown, with details about resulting pups. mental Figure 1C). We also detected PML-RARA protein in this leukemia sample, although we were unable to detect PML-RARA protein in bone marrow, spleen, or CFU-granulocyte/CFU-macrophage (CFU-G/CFU-M) cells from healthy 8-week-old mPML-fPR or mCG-PR mice (Supplemental Figure 1D and data not shown).

PML-RARA activity causes a microstippled pattern of native Pml protein in the nucleus (17). We observed this pattern of Pml nuclear localization in $m \mathrm{CG}-\mathrm{PR}^{+/-}$and $\mathrm{LysM}-\mathrm{Cre}^{+/-} \times \mathrm{mPML}-\mathrm{PRflox}{ }^{+/-}$ splenic PMNs but not in wild-type or $m P M L-P R f_{l o x^{+/-}}$spleen PMNs (Supplemental Figure 1E). After tamoxifen treatment, we also observed microstippling of nuclear Pml in $\mathrm{ERT2}_{2} \mathrm{Cre}^{+/-} \times \mathrm{mPML}$ $P R f_{l o x^{+/-}}$spleen PMNs (Supplemental Figure 1E).

Collectively, these data show that the MPML-fPR allele results in $P m l$ haploinsufficiency, that PML-RARA is expressed after exposure to Cre recombinase, and that $P M L-R A R A$ has the expected effects on cellular functions.

Titrating floxing in vivo. Consistent with prior studies by Hayashi et al., ERT2-Cre activity resulted in dose-dependent floxing of the LoxP-flanked Neo cassette (22). After 5 doses of tamoxifen at $4 \mathrm{mg}$ per dose i.p., we observed approximately $20 \% \mathrm{mPML}-\mathrm{fPR}$ alleles in a wide range of tissues by Southern blotting (data not shown). Because large amounts of DNA are required for this technique, we developed a qPCR-based assay, using primers that span the LoxP Neo cassette ("Flox" primer pair amplified the floxed locus only) or that span the first LoxP site ("Neo" primer pair amplified the native, unfloxed locus only) (Figure 2A and Supplemental Figure 2A). Amplification of flox and neo primer pairs was normalized to amplification across the PML-RARA junction ("PR" primer pair amplified the mPML-PRflox allele). By titrating genomic DNA from mPML-fPR and $m P M L-P R f l o x^{+/-}$spleen cells we could calculate the percentage of floxed alleles within a population (Supplemental Figure 2A). Amplification of a genomic DNA mixture containing a population of $10 \%$ or $90 \%$ mPML-fPR alleles yielded consistent results across multiple experiments (Supplemental Figure 2B). These samples were amplified during each of the subsequent experiments and were used to calibrate floxing efficiency. Using this approach, we confirmed a dose-dependent increase in the MPML-fPR allele in bone marrow from $E R T 2-\mathrm{Cre}^{+/-} \times m P M L-P R$ flox $^{+/-}$mice: after 1, 5, and 10 doses of $4 \mathrm{mg}$ i.p. tamoxifen, we observed approximately $10 \%, 30 \%$, and $95 \%$ floxed alleles, respectively (Supplemental Figure 2C).
Early effects of PML-RARA on hematopoiesis. Despite the significant embryonic toxicity observed with the MPML-PRflox transgene when crossed with $\mathrm{Mx}$-Cre or CMV-Cre, we observed very little acute hematopoietic toxicity in ERT2-Cre ${ }^{+/-} \times m P M L-P R f l o x^{+/-}$ mice after tamoxifen treatment. After 10 doses of i.p. tamoxifen (4 mg each, administered in twice weekly doses), we evaluated peripheral blood counts and bone marrow progenitor compartments in 4 ERT2-Cre ${ }^{+/-} \times m P M L-P R f_{l o x^{+/-}}$mice (these mice all achieved more than $95 \%$ floxing in their bone marrow cells) and their littermate controls. Tamoxifen treatment did not acutely alter total peripheral blood white blood cell numbers, hemoglobin levels, platelets, or the percentage of lymphocytes, granulocytes, or monocytes (data not shown). Evaluation of bone marrow cells revealed a small decrease in $\mathrm{Kit}^{+} \mathrm{Lin}^{-} \mathrm{Sca}^{+}$(KLS) cells in mPML-fPR mice ( $1.3 \%$ vs. $2.5 \%$ of lineage-negative cells; $P=0.09$ ) but no other significant changes in progenitor populations (Figure 3B). Bone marrow cells were cultured in methylcellulose containing IL-3, IL-6, and SCF, and CFUs were assessed after 1 week. mPML-fPR did not significantly alter total CFUs or the immunophenotype of these cells after myeloid differentiation (Figure 3, C and D). Short pulses of $6 \mathrm{mg}$ tamoxifen (given i.p. every other day for 10 days) resulted in $75 \%$ floxing in bone marrow and spleen cells and a nonsignificant trend toward decreased CFUs and CFU-Ms (Supplemental Figure 3, A and B). Exposure to 4-hydroxytamoxifen in vitro caused a modest decrease in total CFUs and CFU-Ms, but there was no change in total CFUs arising in methylcellulose with G-CSF alone (Figure 3E and Supplemental Figure 3C). Surprisingly, these data suggest that acute exposure of hematopoietic cells to PML-RARA (at a dose governed by the $P m l$ locus) is minimally toxic to early progenitor populations, leaving the hematopoietic compartments largely unperturbed.

However, Cre-dependent activation of MPML-PRflox resulted in dysregulation of hematopoietic self-renewal. After 10 doses of tamoxifen (4 mg i.p. twice weekly), bone marrow cells from ERT2-Cre ${ }^{+/-} \times m P M L-P R f l o x^{+/-}$mice could be serially passaged for more than 6 weeks (Figure 4B). The self-renewal phenotype was completely dependent on floxing, since the floxed allele frequency increased during replating and represented $100 \%$ of the alleles after 4 weeks of culture (Figure 4C). Outgrowth of the $\mathrm{MPML}-\mathrm{fPR}$ allele occurred whether the starting bone mar- 
A

B
Methylcellulose

10,000 cells/plate
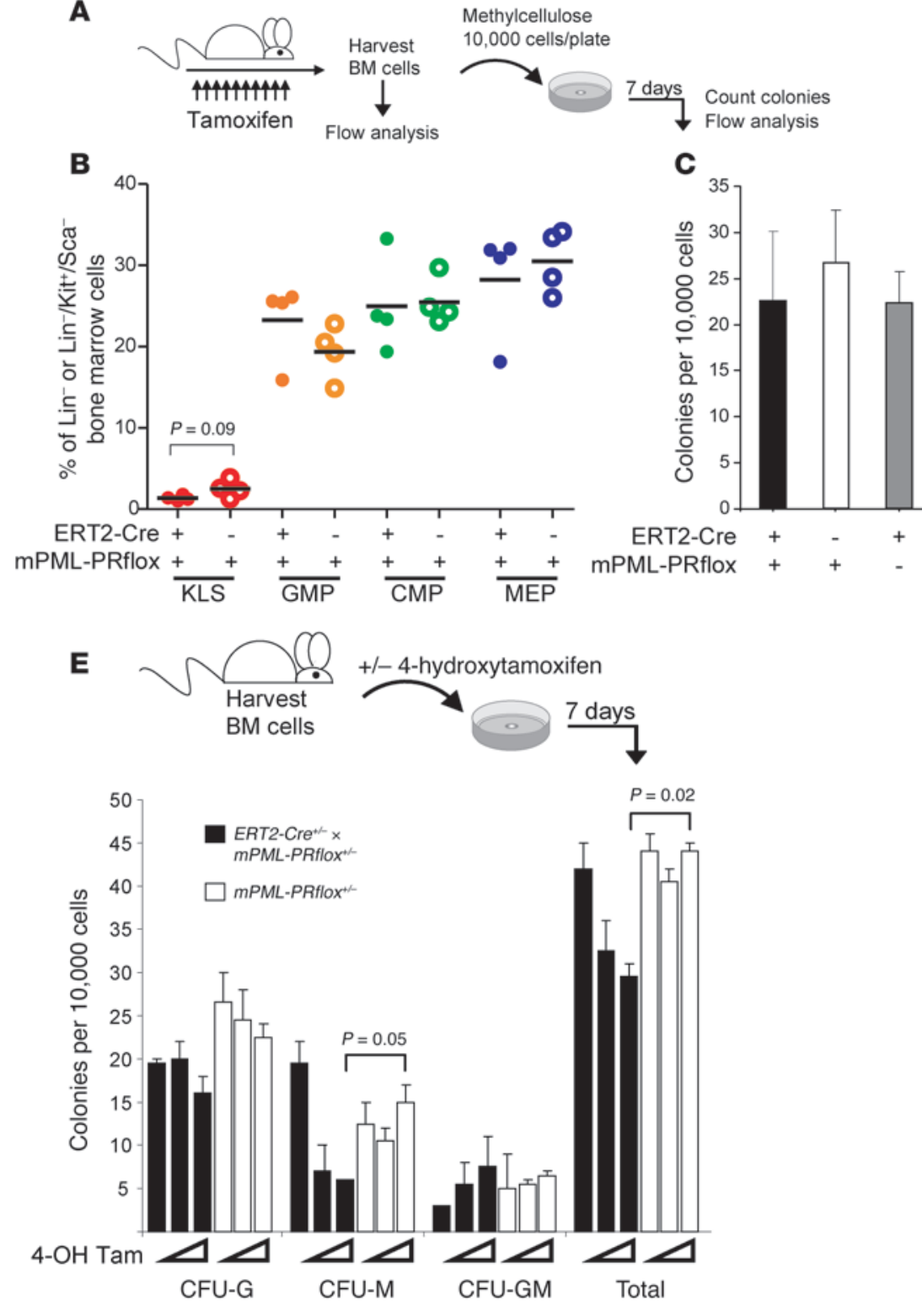

C

C
D 
A

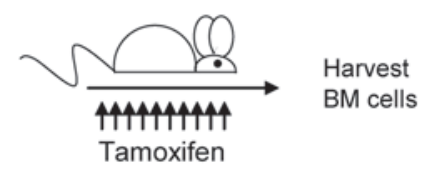

Methylcellulose

10,000 cells/plate
B
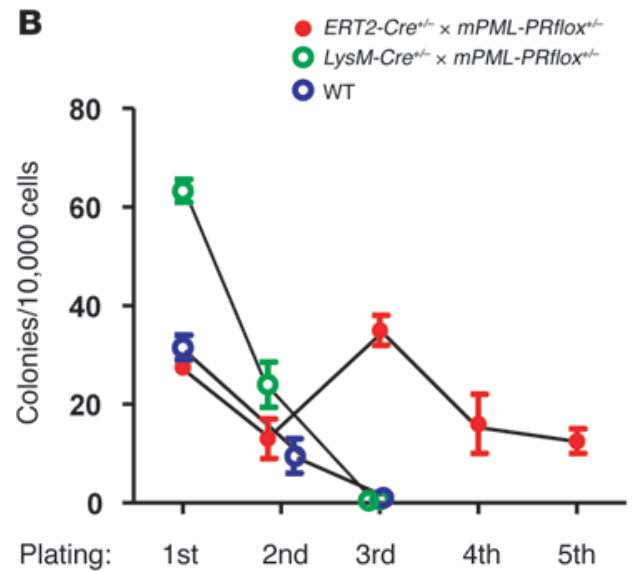

C

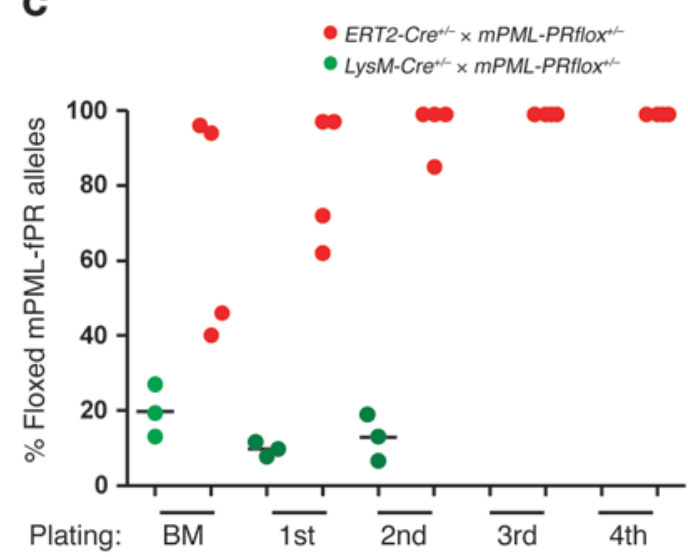

Figure 4

mPML-fPR leads to inappropriate ex vivo self-renewal of bone marrow CFUs. (A) Experiment schema showing that $E R T 2-C r e^{+/-} \times m P M L-$ $P R f l^{+/-}$mice and littermate controls were treated with tamoxifen (4 mg i.p. twice weekly for 10 doses). Bone marrow cells were plated in methylcellulose containing IL-3, IL-6, and SCF. At 7-day intervals, colony numbers were counted and cells were harvested and replated as indicated. (B) mPML-PRflox leads to inappropriate self-renewal when intercrossed with ERT2-Cre but not LysM-Cre. Representative results are from 2 experiments. Data points represent average and SD from 4 individual mice. (C) The mPML-fPR allele was associated with continued CFU activity in ERT2-Cre, but not LysM-Cre, bone marrow. The percentage of mPML-fPR alleles was assessed at each time point in B using qPCR. Data points represent results from individual mice. Horizontal bars are the median value.

founder mPML-PRflox lines and their littermate controls. After 18 months of observation, we observed leukemia in only 1 LysM$\mathrm{Cre}^{+/-} \times m P M L-P R$ flox $^{+/-}$mouse (on day 481; Figure 5, A and B). Leukemic spleen cells expressed a primitive myeloid phenotype: $\mathrm{cKit}^{+} \mathrm{Sca}^{+} \mathrm{CD} 34^{-} \mathrm{CD} 16^{+} \mathrm{Gr} 1^{\mathrm{dim}}$. Analysis of this mouse identified 90\% and 95\% mPML-fPR alleles in the peripheral blood and spleen cells, respectively. Interestingly, this mouse presented with a white blood cell count of 3,600 cells/ $\mu$ l and only modest splenomegaly (460 mg spleen weight). These spleen cells reconstituted leukemia in 5 adoptively transferred B6 mice, whose leukemic spleens contained 100\% floxed alleles (Figure 5, C-E).

Unexpectedly, the peripheral blood and spleen cells at 18 months in nonleukemic mice demonstrated no consistent expansion of a floxed population or evidence of myeloproliferation (Figure 5, A, $\mathrm{F}$, and G). Analysis of $5 \mathrm{mPML}-\mathrm{PR}$ flox ${ }^{+/-}$mice at 18 months showed no evidence of the floxed allele in the absence of Cre recombinase (data not shown). Regardless, these data confirm that the mPMLfPR transgene, activated in the LysM cell population, is capable of causing myeloid leukemia, albeit rarely.

In contrast, after limited exposure to tamoxifen in ERT2-Cre $\mathrm{CR}^{+/-}$ mice, we observed that MPML-fPR caused a competitive advantage within hematopoietic cells in vivo. We treated 2 cohorts of mice (comprised of mice from 2 different founder mPML-PRflox lines and their littermate controls) with $1(n=6)$ or $5(n=4)$ doses of tamoxifen ( $4 \mathrm{mg}$ i.p. twice weekly) and serially assessed the percentage of mPML-fPR alleles within the peripheral blood. Acquisition of the mPML-fPR allele did not alter peripheral blood counts during 6 months of evaluation (Figure 6B and data not shown). How- ever, we observed a marked increase in the percentage of peripheral blood mPML-fPR alleles over this same time period, regardless of the initial efficacy of floxing (Figure 6, C and D). A third cohort of ERT2-Cre ${ }^{+/-} \times m P M L-P R f l o x^{+/-}$mice were followed without tamoxifen treatment. Minimal floxing was detected in the peripheral blood of these mice, showing that leakiness of ERT2-Cre during adult life could not account for the expansion of mPML-fPR-positive cells after tamoxifen treatment (Figure 6D).

This competitive advantage continued through 18 months of age, when the mice were sacrificed. In bone marrow and spleen cells, the mPML-fPR allele comprised more than 95\% of mPML-PRflox alleles in nearly all mice treated with 1 or 5 doses of tamoxifen (Figure 6E and data not shown). The relative percentage of bone marrow floxed alleles at 18 months correlated with prior observations in peripheral blood cells; the 2 mice that did not achieve more than 95\% floxed alleles in the bone marrow had the lowest percentage of peripheral blood floxed alleles from the beginning of the experiment (mouse 11941 and 11933). Again, this expansion of mPMLfPR-positive cells was not associated with myeloproliferation; the mice did not develop splenomegaly or exhibit increased myeloid cells in the bone marrow or spleen (Figure 6F and Supplemental Figure 4). One mouse (mouse 11401) developed a cutaneous infiltrate reminiscent of the cutaneous MRP8-PML-RARA phenotype and was sacrificed on day 249 (data not shown) (6). One mouse (11930) developed splenomegaly. This was associated with a transplantable leukemia that appeared unrelated to mPML-fPR; bone marrow and spleen cells contained only $10 \%$ floxed alleles and did not contain detectable amounts of PML-RARA protein (Figure 6E, 


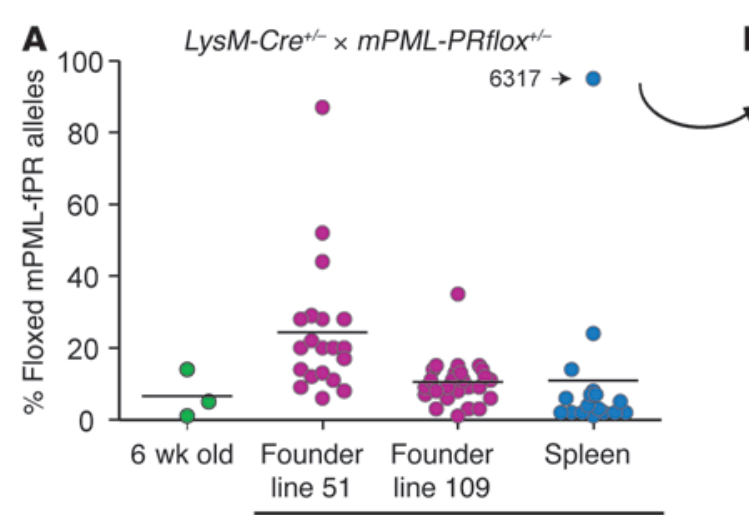

18 months old

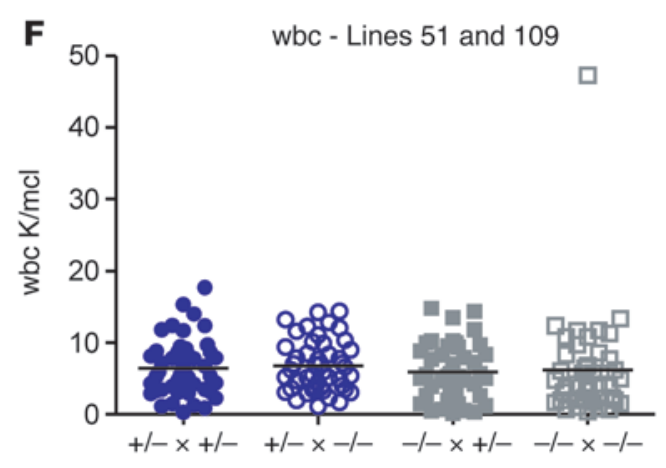

Genotype LysM-Cre $\times$ mPML-PRflox
D $\quad$ E
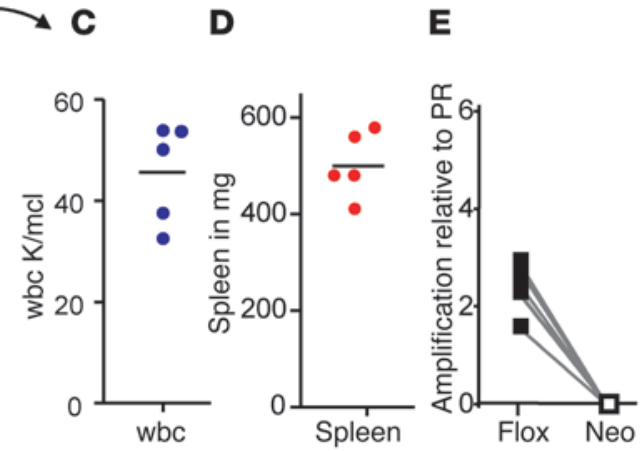

Secondary transplant

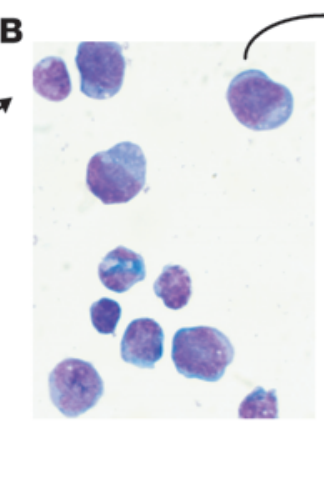

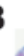

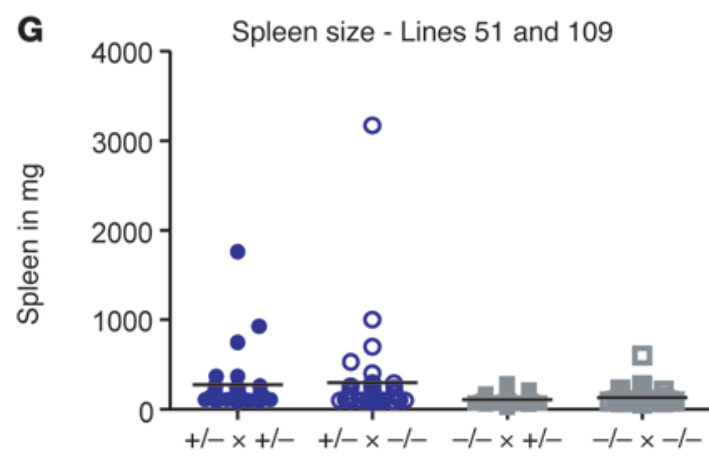

Genotype LysM-Cre $\times$ mPML-PRflox

Figure 5

Rare leukemia in LysM-Cre $\times$ mPML-PRflox mice. (A) A tumor watch of LysM-Cre+-- $\times$ mPML-PRflox ${ }^{+/-}$mice $(n=54)$ resulted in leukemic mouse (mouse 6317, founder line 51) with $90 \%$ and $95 \%$ peripheral blood and spleen mPML-fPR alleles, respectively. Nonleukemic mice were evaluated at 18 months and showed little evidence of expanded mPML-fPR alleles in either peripheral blood or spleen. (B) Spleen cell cytomorphology from mouse 6317, stained with Wright-Giemsa (original magnification, $\times 1,000$ ). (C-E) Spleen cells from mouse 6317 were transplanted into nonirradiated B6 mice $(n=5)$. In 6 weeks all mice were moribund, with (C) elevated white blood cell counts and (D) splenomegaly. (E) qPCR evaluation of the spleen cells in moribund secondary recipients revealed $100 \% \mathrm{mPML}-\mathrm{fPR}$. (F and $\mathbf{G})$ Analysis of $L y s M-C r e^{+/-} \times \mathrm{mPML}^{-P R f l o x^{+/-}}$mice and littermate controls at 18 months revealed normal $(\mathbf{F})$ white blood cell and $(\mathbf{G})$ spleen sizes, with rare evidence of splenomegaly in $L y s M-C r e+-$ mice, regardless of mPML-PRflox genotype. Data points represent results from individual mice. Horizontal bars are the median value.

Supplemental Figure 1D, and data not shown). These data confirm that mPML-fPR provides a competitive advantage in hematopoietic cells in vivo as well as in vitro. Unlike prior models of APL (but similar to human APL), the competitive advantage of mPMLfPR was not associated with myeloproliferation, and homeostatic feedback regulation of myeloid compartment size was preserved.

PML-RARA-dependent leukemia has not yet been observed in $E R T 2-\mathrm{Cr}^{+/-} \times m P M L-P R f_{l o x^{+/-}}$mice. An additional 12 mice were treated with 5 doses of tamoxifen and were followed for 15 months. One mouse became ill with myelodysplastic-like disease characterized by pancytopenia, a normal spleen $(150 \mathrm{mg})$, and an infiltrate of $\mathrm{Gr}^{+} \mathrm{CKit}^{+} \mathrm{CD} 11 \mathrm{~b}^{+}$cells $(79 \%)$ in the bone marrow, consisting predominantly of differentiated PMNs with few blasts (data not shown). CD 45. $2^{+}$cells engrafted 2 sublethally irradiated CD45.1 recipients but did not recapitulate the proband's disease (data not shown).

To determine whether the self-renewal phenotype was related to alterations in cell cycle or apoptosis, we examined BrdU uptake in ERT2-Cre ${ }^{+/-} \times m P M L-P R f l o x^{+/-}$cells, both in vitro and in vivo. We observed no difference in BrdU uptake after in vivo tamoxifen or after acute exposure to 4-hydroxytamoxifen in vitro. We examined unmanipulated total bone marrow cells and bone marrow cells grown ex vivo in liquid culture containing IL-3, IL-6, and SCF, liquid culture containing G-CSF and SCF, or methylcellulose plating experiments containing the IL-3, IL-6, and SCF (data not shown). We examined annexin $\mathrm{V} /$ propidium iodine staining under similar conditions and identified no differences in the frequency of apoptotic cells based on genotype or tamoxifen exposure (data not shown). This suggests that the self-renewal phenotype may occur either through non-cell cycle/apoptotic pathways or that only a small subset of cells may be affected.

To validate the self-renewal phenotype in vivo and to define the compartment affected by mPML-fPR, we performed both competitive and noncompetitive transplantation experiments. Initial competitive transplantation of bone marrow from $E R T 2-\mathrm{Cre}^{+/-} \times m P M L$ PRflox ${ }^{+/}$mice treated with 10 doses of tamoxifen was confounded by an early engraftment defect and wide variability in subsequent competitive expansion (Supplemental Figure 5A). We observed a similar engraftment defect in bone marrow cells from $\mathrm{ERT2}-\mathrm{Cre}^{+/-}$mice treated with tamoxifen, suggesting that this phenotype was caused by Cre toxicity to stem cells, which has been observed by others, and may partially explain the decrease in KLS cells seen in Figure 3B (23). We also observed a consistent competitive advantage associated with 
A

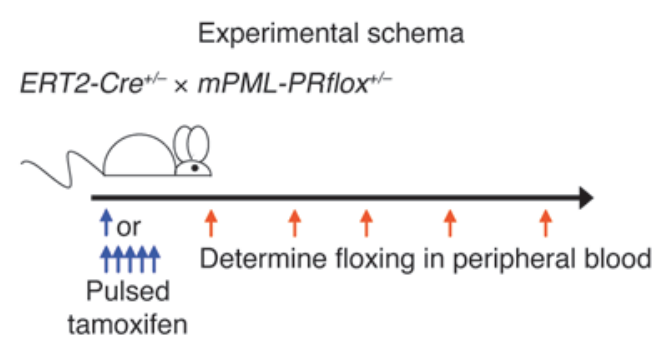

C
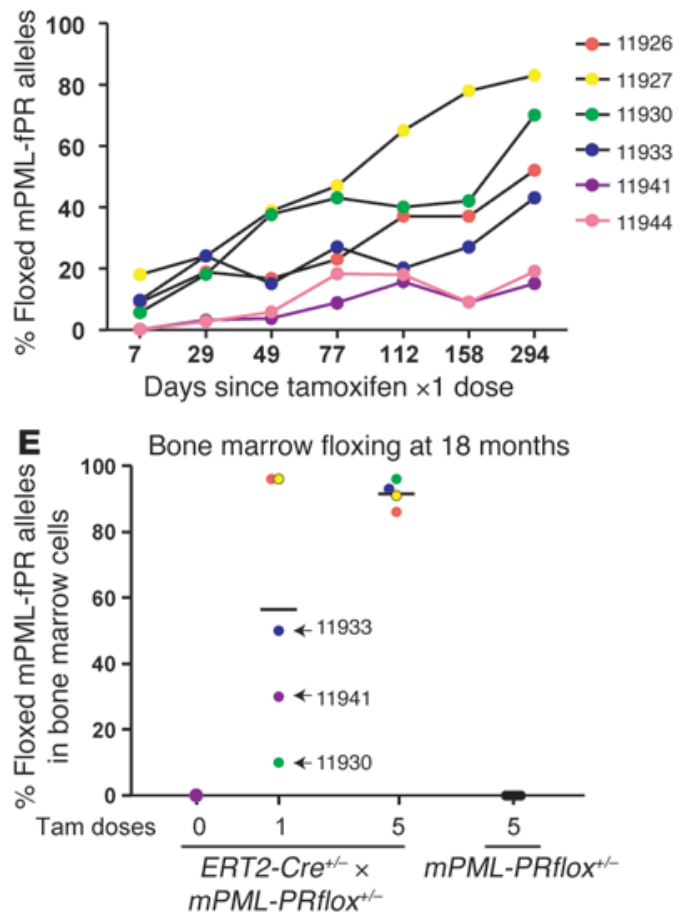

B

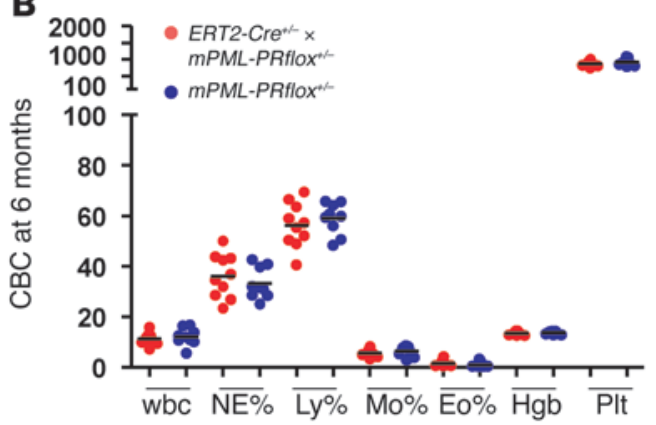

D
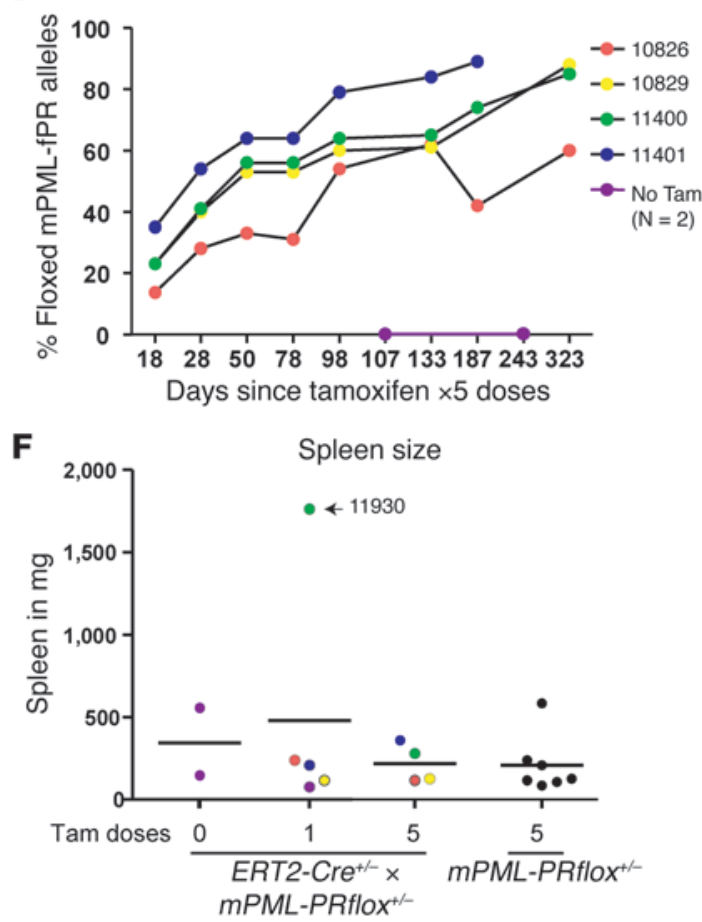

Figure 6

Expansion of mPML-fPR alleles after pulsed tamoxifen. (A) Experimental schema showing ERT2-Cre+/- $\times m P M L-P R f l o x^{+/-} \mathrm{mice}^{-}$and $m P M L-$ $P R f x^{+-}$littermate controls were treated with 0,1 , or 5 doses of tamoxifen (4 mg i.p.). (B) mPML-fPR did not alter peripheral blood counts at 6 months. NE, neutrophil; Ly, lymphocytes; Mo, monocytes; Eo, eosinophils; Hgb, hemoglobin; PIt, platelet. (C and D) After the initial pulse of tamoxifen, the percentage of mPML-fPR alleles in peripheral blood increased and was measured using qPCR at indicated time points. Numbers next to colored dots indicate specific mice. (E) At 18 months, the percentage of mPML-fPR alleles was assessed in bone marrow cells. (F) Expansion of mPML-fPR alleles was not associated with splenomegaly. Data represent results from individual mice at indicated time points. Horizontal bars are the median value.

$P m l$ haploinsufficiency in $m P M L-P R f l o x^{+/-}$bone marrow in both the granulocyte and lymphocyte compartments, consistent with prior observations that $P m l$ haploinsufficiency shortens $\mathrm{mCG}-\mathrm{PR}$ leukemic latency (Supplemental Figure 5C, ref. 11, and data not shown).

We observed a competitive expansion of ERT2-Cre ${ }^{+/-} \times m P M L-$ $\mathrm{PRflox}^{+/-}$bone marrow cells after competitive and noncompetitive transplantation when donor bone marrow was transplanted prior to tamoxifen exposure (Figure 7). After confirming engraftment of competitor and donor cells, we administered 5 doses of tamoxifen (posttransplant recipients tolerated i.p. tamoxifen poorly, and we administered the drug by gavage). During 16 weeks of follow-up, we observed a consistent expansion of donor CD45.2+ peripheral blood cells in ERT2-Cre ${ }^{+/-} \times m P M L-P R$ flox $^{+/-}$recipients but not in $E R T 2-\mathrm{Cre}^{+/-}$recipients (Figure 7, A and B). Similarly, after noncom- petitive transplantation of total bone marrow from $\mathrm{ERT2}-\mathrm{Cre}^{+/-} \times$ $m P M L-P R f^{\prime} x^{+/-}$donors, we observed a consistent expansion of cells with mPML-fPR alleles (Figure 7C). More robust expansion of PML-RARA cells in noncompetitive transplants compared with that in competitive transplants is partly explained by the fact that only a portion $(\sim 40 \%)$ of the CD $45.2^{+}$donor cells were floxed in the competitive studies (Figure 7, A and C, and data not shown).

Competitive expansion occurred across multiple hematopoietic lineages, suggesting that mPML-fPR affects a stem cell compartment with multilineage potential (Supplemental Figure 6, A-D). As expected, we observed initial peripheralization of donor $\mathrm{Gr}^{+}$and $\mathrm{Nk} 1.1^{+}$cells from both ERT2-Cre $e^{+/-} \times m P M L-P R$ flox $^{+/-}$and ERT2$\mathrm{Cr}^{+/-}$donor cells, while peripheralization of $\mathrm{CD} 19^{+}$and $\mathrm{CD}^{+}$ donor cells occurred more slowly (Supplemental Figure 6). After 
A Competitive transplant 1:3 then tamoxifen $\times 5$ doses ERT2-Cre ${ }^{+/} \times$mPML-PRflox ${ }^{+/-}$

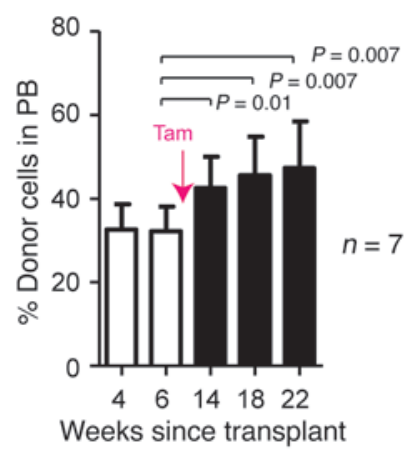

Competitive transplant 1:3 then tamoxifen $\times 5$ doses ER-T2Cre

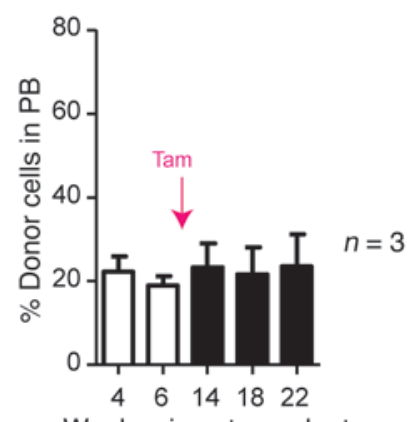

Weeks since transplant
C Transplant total marrow then tamoxifen $\times 5$ doses ERT2-Cre $+/-\times$ mPML-PRflox ${ }^{+/-}$

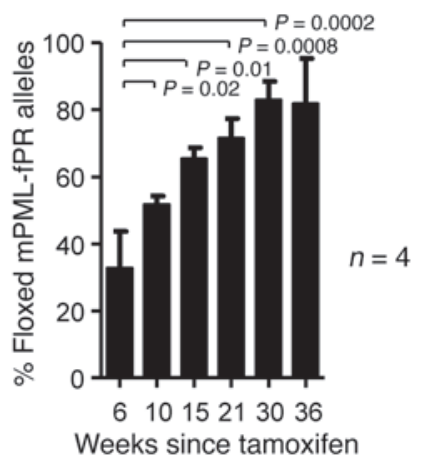

\section{Figure 7}

Competitive and noncompetitive transplantation. Bone marrow from indicated donor mice (CD45.2) was transplanted with competitor bone marrow (CD45.1) into lethally irradiated recipients (CD45.1). After engraftment, recipients were treated with the indicated doses of tamoxifen (3 $\mathrm{mg}$ by gavage). At the indicated time points, peripheral blood (PB) was assessed by flow cytometry. (A) Assessment of ERT2-Cre ${ }^{+/-} \times$ $m P M L-P R f l o x^{+/-}$donor bone marrow cells. (B) Assessment of $E R T 2-C_{r e}^{+/-}$donor bone marrow cells. (C) Assessment of ERT2-Cre ${ }^{+/-} \times m P M L-$ $P R f_{l o X^{+-}}$donor bone marrow cells. Results represent average and SD from indicated numbers of mice in each cohort.

tamoxifen, we observed expansion of ERT2-Cre ${ }^{+/-} \times m P M L-P R f l o x^{+/-}$ donor cells within $\mathrm{CD}_{1} 9^{+}, \mathrm{Nk} 1.1^{+}$, and $\mathrm{CD}^{+}$lymphoid compartments but no expansion of ERT2-Cre ${ }^{+/-}$donor cells (Supplemental Figure 6, B-D and F-H). By 14 weeks after transplant, the percentage of donor ERT2-Cre ${ }^{+/-}$cells declined in $\mathrm{Gr}^{+}$myeloid cells and remained constant with respect to the percentage of total donor cells in the peripheral blood at 6 weeks (Supplemental Figure 6E). In contrast, the percentage of $\mathrm{Gr}^{+} \mathrm{ERT}_{2}-\mathrm{Cre}^{+/-} \times \mathrm{mPML}-\mathrm{PRflox} \mathrm{x}^{+/-}$ donor cells remained significantly higher than the percentage of total donor cells in the peripheral blood at 6 weeks (Supplemental Figure 6A). As before, expansion of mPML-fPR-positive cells was not associated with myeloproliferation; recipient mice maintained normal CBCs and peripheral blood differential counts throughout the experiment (data not shown). These data provide further evidence that $P M L-R A R A$ results in competitive expansion across multiple hematopoietic lineages, without disturbing the homeostatic regulation of myeloid or lymphoid compartments.

\section{Discussion}

PML-RARA expression has been shown to lead to leukemia in multiple prior mouse models (reviewed in refs. 3, 4). This leukemia is typically associated with antecedent myeloproliferation (increased promyelocytes and CFUs), requires cooperating progression mutations, and generally presents with high white blood cell counts ( 7 , $8,24,25)$. Because human APL is characterized by low white blood cell counts and an acute onset without antecedent myeloproliferation (1), we sought to more accurately model human APL by placing PML-RARA under the regulatory control of the Pml locus, in the setting of somatic acquisition of expression and $P m l$ haploinsufficiency. Our observations that $P M L$ and $R A R A$ are not grossly dysregulated in $\mathrm{t}(15 ; 17)$ human leukemic bone marrow samples suggest that the translocation does not dramatically alter the expression of either gene and that accurate, somatic expression of PML-RARA is likely to be important.

Our data show that adult acquisition of hematopoietic PML-RARA expression leads to a competitive advantage that allows PML-RARAexpressing cells to expand more efficiently than their wild-type counterparts both in vitro and in vivo. However, this expansion is not associated with evidence of myeloproliferation or dysregulation of hematopoietic homeostasis (mice had normal progenitor numbers, CFUs, and normal CBCs and differentials during followup). However, LysM-Cre (which is predominantly active in mature PMNs and macrophages) (20) did not recapitulate this self-renewal phenotype, raising the possibility that PML-RARA cannot reprogram terminally differentiated myeloid cells to self-renew.

The in vivo self-renewal phenotype was recapitulated after competitive and noncompetitive transplantation (i.e., it was cell autonomous), and it was associated with both lymphoid and myeloid expansion but normal CBCs and differential counts. Collectively, these experiments demonstrate that PML-RARA affects a multipotent progenitor compartment. These results are supported by our recent findings in $\mathrm{mCG}-\mathrm{PR}$ mice, in which we have demonstrated that PML-RARA is expressed and functional in the KLS compartment (Welch et al., unpublished observations). Both results contradict the current paradigm, which suggests that PML-RARA transforms a committed myeloid precursor (i.e., a promyelocyte) by being expressed at that stage of development (26-29). This is clearly not the case. It will be important to determine whether the maturation arrest results from development-specific cooperating factors (i.e., neutrophil elastase $[E L A N E]$ ) or from cooperating mutations that display myeloid selectivity (i.e., mutations that occur in genes with myeloid-restricted expression) (17).

The leukemic penetrance of mPML-PRflox was very low in both LysM-Cre and ERT2-Cre intercrosses. This represents the sixth mouse model generated using the same PML-RARA cDNA. Each of these prior models have resulted in very different leukemic penetrance, ranging from zero ( $c$ Fes and CD11b) to nearly complete (mCG-PR and MRP8-PR), suggesting that the dose and timing of PML-RARA expression do significantly alter the susceptibility of mice to leukemia development (Welch et al., unpublished observations) (7-9, 30-32).

The model described here differs from the prior models in several ways. Importantly, the expression of PML-RARA mRNA from the $P m l$ locus is even lower than that measured from the mCG-PR 
mice, which is in turn much lower than the expression in hCG-PR transgenic animals. Consistent with this, we have recently found that the expression pattern of $P M L$ is very different from that of CTSG and MRP8 in both mice and humans; it is much lower with no dynamic induction during promyelocyte maturation (Welch et al., unpublished observations). Our group has previously shown that reduced dose levels of PML-RARA are associated with less neutrophil elastase-dependent myeloid toxicity (17). However, the dose of PML-RARA expression obtained in mCG-PR mice is still associated with myeloid proliferation, reminiscent of "rebound" after recovery from cytotoxic therapy. In contrast, after acute exposure to mPML-fPR, we observed essentially no hematologic toxicity and no evidence for myeloproliferation (normal progenitor numbers, normal differential counts, and normal CFU numbers). This decrease in toxicity and low leukemic penetrance makes this model an attractive system for testing mutational cooperativity. Studies are ongoing to assess the effects of JAK1 V658F (33) and radiation in these mice (34). Because antecedent myeloproliferation is not observed in APL patients, we suggest that the myeloproliferation and the increased penetrance observed in $\mathrm{mCG}-\mathrm{PR}$ mice may partially reflect the hematologic toxicity of overexpressed PML-RARA and the resultant early hematopoietic expansion that may occur as a consequence. These results suggest that $P M L-R A R A$, when expressed from the $P m l$ locus, is not a "potent" oncogene and raise questions about the true penetrance of APL in people who spontaneously acquire $t(15 ; 17)$ in hematopoietic cells.

Finally, these data suggest a mechanism of initial $t(15 ; 17)$ activity. PML-RARA is necessary but not sufficient to cause leukemia in mice (in mouse models there is substantial leukemic latency that can be shortened by radiation, alkylator treatment, or cooperation with other known leukemia progression mutations) $(3,35)$. However, human APL is characterized by an acute presentation, often in extremis $(1)$. How does $t(15 ; 17)$ lead to an acute disease if it requires additional cooperating mutations? The results presented here and other data suggest that $t(15 ; 17)$ leads to a competitive advantage that allows for expansion of $\mathrm{t}(15 ; 17)$-positive cells (36). This increases the probability of acquiring additional mutations in $\mathrm{t}(15 ; 17)$-positive cells, but it does not lead to myeloproliferation that could cause a chronic hematopoietic disorder. PML-RARA increases the number of self-renewing progenitors, but homeostatic feedback is lost only after critical secondary mutations are acquired. Further studies will be required to fully validate this model, but the data presented here have uncovered substantial differences from previous models with less physiologic designs. Once again, this study points out the importance of accurately reproducing every aspect of oncogene activation to mimic the evolution of human cancers.

\section{Methods}

Expression array profiling. Human AML and normal hematopoietic cell expression profiling has been previously described (37). In brief, bone marrow aspirates were obtained from patients that gave informed consent. RNA was prepared from the unfractionated snap-frozen cell pellets and profiled using the Affymetrix Human Genome U133 Plus 2.0 Array GeneChip microarrays (Affymetrix) at the Siteman Cancer Center Molecular and Genomic Analysis Core Facility (38).

Mice. The mPML-PRflox transgene was generated as described in Figure 2. PML-RARA cDNA was cloned downstream of a loxp-PGK-neo-stop-loxp cassette and then flanked by the $4.5-\mathrm{kb}$ XbaI-EcoRI region upstream of $P m l$ exon 1 and by the 4-kb EcoRI-ClaI fragment downstream of Pml exon 1.
This construct was electroporated into B6-Blu ES cells made in the Siteman Cancer Center Embryonic Stem Cell Core (http://escore.im.wustl.edu/). Two independent targeted clones (numbered 51 and 109) were identified by Southern blotting and injected into B6 blastocysts. Chimeric male offspring were identified by LacZ activity in the peripheral blood, and the targeted locus in both clones was transmitted through the germline of B6 mice. Intercrosses were performed as described. Mx-Cre (B6.Cg-Tg[Mx1-cre $] 1 \mathrm{Cgn} / \mathrm{J})$,

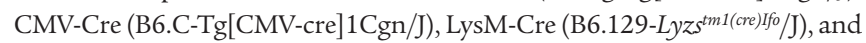
ERT2-Cre (B6.Cg-Tg[cre/Esr1]5Amc/J) mice were obtained from The Jackson Laboratory, and all were otherwise on a B6 background. Activation of ERT2-Cre was performed by i.p. injection or gavage of tamoxifen dissolved in sterile corn oil (Sigma-Aldrich) as previously described (22). In wild-type B6 mice, $6 \mathrm{mg}$ i.p. tamoxifen proved to be dose limiting, and we thus typically administered $4 \mathrm{mg}$ tamoxifen. Peripheral blood counts were accessed as indicated (Hemavet 950, Drew Scientific Group).

Competitive repopulation was performed by retro-orbital injection of $1 \times 10^{6}$ total bone marrow cells into lethally irradiated Ly5.1 recipients (CD45.1) that received 1,100 cGy total body irradiation (Mark 1 Cesum ${ }^{137}$ irradiator, JL Shepherd) 24 hours prior to transplantation.

The Washington University Institutional Review Board approved the collection and molecular characterization of bone marrow cells collected from AML patients and from normal subjects. The Washington University Animal Studies Committee approved all animal experiments.

Hematopoietic progenitor assay. After lysis of red blood cells (ACK buffer: $0.15 \mathrm{M} \mathrm{NH}_{4} \mathrm{Cl}, 10 \mathrm{mM} \mathrm{KHCO}_{3}, 0.1 \mathrm{mM} \mathrm{Na}_{2}$ EDTA), bone marrow cells were counted using a hemocytometer and plated in duplicate in $1.1 \mathrm{ml}$ of methylcellulose medium containing IL-6, IL-3, and SCF (MethoCult M3534, StemCell Technologies) (36). Cells were plated at $8.3 \times 10^{3}$ cells $/ \mathrm{ml}$. Colonies with more than 30 cells were counted on day 7 . Cells were collected in warm DMEM, washed, and counted. Cells were then replated at the same density. This process was repeated for 6 weeks or until colony formation failed.

Flow cytometry. After lysis of red blood cells, peripheral blood, bone marrow, or spleen cells were treated with anti-mouse CD16/32 (eBioscience, clone 93) and stained with the indicated combinations of the following antibodies: FITC- $\alpha$ Gr1 (BD Pharmingen, clone RB6-8C5); PE- $\alpha$ CD11b (eBioscience, clone M1/70); PE- $\alpha \mathrm{CD} 115$ (eBioscience, clone AFS98); FITC- $\alpha \mathrm{CD} 34$ (BD Pharmingen, clone RAM34); FITC-NK1.1 (eBioscience, clone PK136); APCacKit (eBioscience, clone 2B8); PE- $\alpha \mathrm{CD} 3$ (BD Pharmingen, clone 145-2C11); PE- $\alpha$ CD19 (eBioscience, clone MB19-1); PE- $\alpha$ B220 (BD Pharmingen, clone RA3-6B2); biotin- $\alpha \mathrm{CD} 34$ (eBioscience, clone RAM34); PercP-Cy5.5- $\alpha \mathrm{Gr} 1$ (eBioscience, clone RB6-8C5); PerCP-Cy5.5- $\alpha$ CD45.2 (eBioscience, clone 104); APC- $\alpha \mathrm{CD} 45.1$ (eBioscience, clone A20); APC- $\alpha \mathrm{Sca}$ (eBioscience, clone D7); APC-Cy7- $\alpha \mathrm{CD} 117$ (eBioscience, clone $2 \mathrm{~B} 8$ ); or lineage cocktail ( $\alpha \mathrm{CD} 3$, CD3e), $\alpha \mathrm{CD} 8$ (clone 53-6.7), $\alpha \mathrm{CD} 4$ (clone L3T4), $\alpha \mathrm{CD} 19$ (clone 1D3), aB220 (clone RA3-6B2), $\alpha$ Gr1 (clone RB6-8C5), or $\alpha$ Terr-119 (clone Ly-76) (all PE-Cy7-conjugated antibodies, eBioscience). Analysis was performed using a FACScan (Beckman Coulter) or Reflection cell sorter (iCyt), and data analyzed using FlowJo, Excel (Microsoft), and Prism 5 (Graphpad).

Western blotting. Whole cell lysates were prepared from spleen cells and NB4 cells in $2 \times$ sample buffer. Lysates from equal numbers of cells were separated on a $10 \%$ NuPAGE Bis-Tris gel (Invitrogen) and transferred onto Hybond C Extra nitrocellulose membrane (Amersham). The membrane was probed with an anti-Pml antibody (1:500; Millipore, clone 16.1-104), a polyclonal antiRARA antibody (a gift from Scott Kogan, UCSF, San Francisco, California, USA) or anti- $\alpha$ Actin (1:1,000; Millipore). Immune complexes were revealed by a peroxidase-conjugated anti-mouse IgG $(1: 10,000$; GE) or conjugated antirabbit IgG (1:2,500; GE) and visualized by chemiluminescence (Pierce).

Immunofluorescence. Spleen cells were fixed in methanol at $-20^{\circ} \mathrm{C}$ and then rehydrated in PBS. Staining was done with $\alpha$-Pml (1:200; Millipore, clone 16.1-104), goat $\alpha$-mouse IgG Alexa Fluor 488 (1:200; Invitrogen) in 
$2 \%$ goat serum, and Vectashield (H-1200, Vector Laboratories). Cells were visualized on a Microphot-SA with Digital Sight imaging (Nikon).

qRT-PCR. Bone marrow and spleen cells were prepared using TRIzOL (Invitrogen). RNA was treated twice with Turbo DNAfree (Ambion). cDNA was prepared using Superscript (Invitrogen) and amplified using QuantiTect SYBR Green (Qiagen) and 7300 Real Time PCR (Applied Biosystems) per manufacture specifications. Primers used were as follows: PML-RARA (ENF903 TCTTCCTGCCCAACAGCAA; ENR962 GCTTGTAGATGCGGGGTAGAG); Pml (mPML-F CAGCCATTGAGACCCAGAGCAG; mPML-R GCAAGGCTTGTAGATGCGGGGTAG); and Gapdb (GAP-F TGCACCACCAACTGCTTAG; GAP-R GGATGCAGGGATGATGTTC).

qPCR. The percentage of floxed alleles was determined after DNA extraction using a DNeasy Blood and Tissue Kit (Qiagen). Genomic DNA was sonicated at $50 \%$ for 30 seconds using a Branson Sonifier 450 (VWR Scientific). Quantitative PCR was performed using QuantiTect SYBR Green (Qiagen) and a 7300 Real Time PCR (Applied Biosystems) per manufacturer specifications. Primers used were as follows: floxed (Floxed2F CCATTTCCCGCCTTCACCAGACCTA; Floxed2R GATCGGGATCGAATTCGGCTTGTACTGA); unfloxed (Floxed2F CCATTTCCCGCCTTCACCAGACCTA; Neo7R GTAGCGCCAAGTGCCCAGC); and PR control (ENF903 TCTTCCTGCCCAACAGCAA; ENR962 GCTTGTAGATGCGGGGTAGAG).

Statistics. Two-sided Student's $t$ test was calculated using Excel (Microsoft). $P$ values $<0.05$ were considered statistically significant. Results with signifi- cant $P$ values are indicated in the text and figures, and the corresponding $P$ values are provided. Nonsignificant $P$ values generally are not included in figures. Data presented with column graphs and error bars represent the average \pm SD. Other data represents results from individual mice.

\section{Acknowledgments}

We thank Scott Kogan (UCSF) for the gift of the rabbit polyclonal anti-RARA antibody; Mieke Hoock for excellent mouse colony management; Nicolas Protopsaltis for expert technical assistance; the Alvin J. Siteman Cancer Center for the use of the High Speed Cell Sorter Core, which provided stem cell analysis; and the Embryonic Stem Cell Core, which generated targeted ES cells. This work was supported by a Leukemia and Lymphoma Society Fellows award (5340-10 to J.S. Welch), NIH grants CA83962 and CA101937, and the Barnes Jewish Hospital Foundation (to T.J. Ley).

Received for publication October 22, 2010, and accepted in revised form January 5, 2011.

Address correspondence to: Timothy J. Ley, Box 8007, 660 South Euclid Ave., Washington University School of Medicine, St. Louis, Missouri 63119, USA. Phone: 314.362.8831; Fax: 314.362.9333; E-mail: timley@wustl.edu.
1. Jurcic JG, Soignet SL, Maslak AP. Diagnosis and treatment of acute promyelocytic leukemia. Curr Oncol Rep. 2007;9(5):337-344.

2. Borrow J, et al. Diagnosis of acute promyelocytic leukaemia by RT-PCR: detection of PML-RARA and RARA-PML fusion transcripts. BrJ Haematol. 1992;82(3):529-540.

3. Kogan SC. Mouse models of acute promyelocytic leukemia. Curr Top Microbiol Immunol. 2007;313:3-29.

4. Westervelt P, Ley TJ. Seed versus soil: the importance of the target cell for transgenic models of human leukemias. Blood. 1999;93(7):2143-2148.

5. Grignani F, et al. PML/RAR alpha fusion protein expression in normal human hematopoietic progenitors dictates myeloid commitment and the promyelocytic phenotype. Blood. 2000;96(4):1531-1537.

6. Brown D, et al. A PMLRARalpha transgene initiates murine acute promyelocytic leukemia. Proc Natl Acad Sci U S A. 1997;94(6):2551-2556.

7. Westervelt $P$, et al. High-penetrance mouse model of acute promyelocytic leukemia with very low levels of PML-RARalpha expression. Blood. 2003; 102(5):1857-1865

8. Grisolano JL, Wesselschmidt RL, Pelicci PG, Ley TJ. Altered myeloid development and acute leukemia in transgenic mice expressing PML-RAR alpha under control of cathepsin $\mathrm{G}$ regulatory sequences. Blood. 1997;89(2):376-387.

9. He LZ, et al. Acute leukemia with promyelocytic features in PML/RARalpha transgenic mice. Proc Natl Acad Sci U S A. 1997;94(10):5302-5307.

10. Liang D, et al. Expressions of Raldh 3 and Raldh4 during zebrafish early development. Gene Expr Patterns. 2008;8(4):248-253.

11. Rego EM, Wang ZG, Peruzzi D, He LZ, CordonCardo C, Pandolfi PP. Role of promyelocytic leukemia (PML) protein in tumor suppression. J Exp Med. 2001;193(4):521-529.

12. Frohling S, Dohner H. Chromosomal abnormalities in cancer. N Engl J Med. 2008;359(7):722-734.

13. Tomasson $\mathrm{MH}$, et al. Somatic mutations and germline sequence variants in the expressed tyrosine kinase genes of patients with de novo acute myeloid leukemia. Blood. 2008;111(9):4797-4808.

14. Purton LE, et al. RARgamma is critical for maintaining a balance between hematopoietic stem cell self-renewal and differentiation. J Exp Med. 2006;
203(5):1283-1293.

15. Chan IT, et al. Conditional expression of oncogenic K-ras from its endogenous promoter induces a myeloproliferative disease. J Clin Invest. 2004; 113(4):528-538.

16. UniGene: Organized View of the Transcriptome. http://www.ncbi.nlm.nih.gov/unigene/. Accessed February 18, 2011.

17. Lane AA, Ley TJ. Neutrophil elastase is important for PML-retinoic acid receptor alpha activities in early myeloid cells. Mol Cell Biol. 2005;25(1):23-33.

18. Ferrucci PF, Grignani F, Pearson M, Fagioli M, Nicoletti I, Pelicci PG. Cell death induction by the acute promyelocytic leukemia-specific PML/RARalpha fusion protein. Proc Natl Acad Sci U S A. 1997; 94(20):10901-10906.

19. Grignani F, et al. The acute promyelocytic leukemia-specific PML-RAR alpha fusion protein inhibits differentiation and promotes survival of myeloid precursor cells. Cell. 1993;74(3):423-431.

20. Clausen BE, Burkhardt C, Reith W, Renkawitz R, Forster I. Conditional gene targeting in macrophages and granulocytes using LysMcre mice. Transgenic Res. 1999;8(4):265-277.

21. Ye M, et al. Hematopoietic stem cells expressing the myeloid lysozyme gene retain long-term, multilineage repopulation potential. Immunity. 2003;19(5):689-699.

22. Hayashi S, McMahon AP. Efficient recombination in diverse tissues by a tamoxifen-inducible form of Cre: a tool for temporally regulated gene activation/inactivation in the mouse. Dev Biol. 2002;244(2):305-318.

23. Higashi AY, et al. Direct hematological toxicity and illegitimate chromosomal recombination caused by the systemic activation of CreERT2. J Immunol. 2009;182(9):5633-5640

24. Welch JS, Klco JM, Varghese N, Nagarajan R, Ley $\mathrm{TJ}$. Rara haploinsufficiency modestly influences the phenotype of APL [published online ahead of print December 29, 2010]. Blood. doi:blood-201008-300087v1-blood-2010-08-300087.

25. Uy GL, Lane AA, Welch JS, Grieselhuber NR, Payton JE, Ley TJ. A protease-resistant PML-RAR $\{$ alpha $\}$ has increased leukemogenic potential in a murine model of acute promyelocytic leukemia. Blood. 2010;116(18):3604-3610.

26. Turhan A, et al. Highly purified primitive hemato- poietic stem cells are PML-RARA negative and generate nonclonal progenitors in acute promyelocytic leukemia. Blood. 1995;85(8):2154-2161.

27. Wojiski S, et al. PML-RARalpha initiates leukemia by conferring properties of self-renewal to committed promyelocytic progenitors. Leukemia. 2009;23(8):1462-1471.

28. Guibal FC, et al. Identification of a myeloid committed progenitor as the cancer-initiating cell in acute promyelocytic leukemia. Blood. 2009; 114(27):5415-5425.

29. Bonnet D, Dick JE. Human acute myeloid leukemia is organized as a hierarchy that originates from a primitive hematopoietic cell. Nat Med. 1997; 3(7):730-737.

30. Zhu J, et al. A sumoylation site in PML/RARA is essential for leukemic transformation. Cancer Cell. 2005;7(2):143-153.

31. Pandolfi PP. Acute myelocytic leukemia. I. Transgenic models of acute myeloid leukemias. In: Hematology 1997: Education Program of the American Society for Hematology. San Diego, California, USA: 1997.

32. Early E, et al. Transgenic expression of PML/RARalpha impairs myelopoiesis. Proc Natl Acad Sci US A. 1996;93(15):7900-7904.

33. Wartman LD, et al. Conserved progression mutations revealed by sequencing a mouse acute promyelocytic leukemia genome. J Clin Invest. 2001; 121(4):1445-1455.

34. Walter MJ, et al. Expression profiling of murine acute promyelocytic leukemia cells reveals multiple model-dependent progression signatures. Mol Cell Biol. 2004;24(24):10882-10893.

35. Funk RK, et al. Quantitative trait loci associated with susceptibility to therapy-related acute murine promyelocytic leukemia in hCG-PML/RARA transgenic mice. Blood. 2008;112(4):1434-1442.

36. Walter MJ, et al. Reduced PU.1 expression causes myeloid progenitor expansion and increased leukemia penetrance in mice expressing PML-RARalpha. Proc Natl Acad Sci U S A. 2005;102(35):12513-12518.

37. Payton JE, et al. High throughput digital quantification of mRNA abundance in primary human acute myeloid leukemia samples. J Clin Invest. 2009; 119(6):1714-1726.

38. Yuan W, et al. Commonly dysregulated genes in murine APL cells. Blood. 2007;109(3):961-970. 